\title{
De-methylation of displacement loop of mitochondrial DNA is associated with increased mitochondrial copy number and nicotinamide adenine dinucleotide subunit 2 expression in colorectal cancer
}

\author{
JINHANG GAO $^{1,2}$, SHILEI WEN ${ }^{1}$, HONGYING ZHOU $^{1}$ and SHI FENG ${ }^{1}$ \\ ${ }^{1}$ Department of Human Anatomy, Academy of Preclinical and Forensic Medicine, West China Medicine College; \\ ${ }^{2}$ Department of Peptides Related to Human Diseases, State Key Laboratory of Biotherapy, \\ West China Hospital, Sichuan University, Chengdu, Sichuan 610041, P.R. China
}

Received November 11, 2014; Accepted July 28, 2015

DOI: $10.3892 / \mathrm{mmr} .2015 .4256$

\begin{abstract}
DNA methylation occurs in the displacement loop (D-loop) region of mammals; however, D-loop regions of certain tumor tissue types were found to be de-methylated. Whether hypomethylation of the D-loop region is involved in the regulation of the mitochondrial DNA (mtDNA) copy number and nicotinamide adenine dinucleotide subunit 2 (ND-2) expressions in colorectal cancer has remained elusive. In the present study, the association between methylation status of the D-loop region, mtDNA copy number and ND-2 expression was investigated in 65 colorectal cancer specimens and their corresponding non-cancerous tissues. In addition, a de-methylation experiment was performed on the Caco-2 colorectal cancer cell line by using 5 -aza-2'-deoxycytidine (5-Aza). The methylation rate of the D-loop region in all 65 colorectal cancer tissues was markedly reduced when compared with that of their corresponding non-cancerous tissues $(13.8$ vs. $81.5 \%$; $\mathrm{P}<0.05)$. Furthermore, the methylation rate of the D-loop region in colorectal cancer tissues was markedly decreased in clinicopathological stages III and IV compared with that in clinicopathological stages I and II (7.1 and $0 \%$ vs. 25 and $16 \%$; $<0.05$ ). In addition, the mean relative mtDNA copy number and ND-2 expression in colorectal cancer tissues were increased compared with those
\end{abstract}

Correspondence to: Dr Shi Feng or Professor Hongying Zhou, Department of Human Anatomy, Academy of Preclinical and Forensic Medicine, West China Medicine College, Sichuan University, 17 3rd Section South Renmin Road, Chengdu, Sichuan 610041, P.R. China

E-mail: jetmork@126.com

E-mail: eaglezhyxzy@163.com

Key words: Caco-2, colorectal cancer, de-methylation, displacement loop, nicotinamide adenine dinucleotide subunit 2, mitochondrial DNA in the corresponding non-cancerous tissues. De-methylation of the D-loop region was associated with an elevated mtDNA copy number and an increased ND-2 expression. Furthermore, the mtDNA copy number and ND-2 expression in Caco-2 cells were significantly increased after 5-Aza treatment. In conclusion, de-methylation of the D-loop region is likely to be involved in the regulation of the mtDNA copy number and ND-2 expression.

\section{Introduction}

Colorectal cancer is one of the most common malignancies and is a major health burden worldwide $(1,2)$. During the past decade, most studies have focused on the roles of nuclear DNA alterations in colorectal cancer, while alterations in mitochondrial DNA (mtDNA) have received a lesser amount of attention. Human mtDNA is a 16,569-base circular double-stranded DNA molecule, which contains genes coding for 13 polypeptides involved in the respiratory chain, 22 transfer RNAs and 2 ribosomal RNAs that are critical for protein synthesis in mitochondria (3). Replication of mtDNA as well as transcription and translation of mtDNA-coding genes are thought to be the major alterations of mtDNA.

Replication of mtDNA induces alterations in the mtDNA content, which is quantified by its copy number. The mtDNA copy number varies from hundreds to $>10,000$ per cell (4). Previous studies have demonstrated that increased mtDNA copy number was closely associated with an increased mortality in advanced gastric cancer patients (5). Furthermore, increased mtDNA content was shown to be associated with a decreased survival rate of patients with tumors $(5,6)$. A previous study by our group demonstrated that the mtDNA copy number was increased in colorectal cancer, and that the mtDNA copy number in clinicopathological stages I and II was significantly higher than that in stages III and IV, which indicated that the mtDNA copy number has a crucial role during the initiation and progression of colorectal cancer (7). Despite the involvement of changes in the mtDNA content in the tumorigenesis of numerous malignancies, the factors 
involved in the alterations of the mtDNA content in colorectal cancer have largely remained elusive.

Alterations in gene transcription and translation may lead to functional deficiency of mitochondria by de-regulating their energy metabolism, leading to excessive generation of free radicals, which trigger programmed cell death (8). Nicotinamide adenine dinucleotide (NADH) is a rate-limiting enzyme of oxidative phosphorylation, and nicotinamide adenine dinucleotide subunit 2 (ND-2) is encoded by mtDNA. Although a previous study by our group has revealed that the expression of ND-2 was increased in colorectal cancer (9), further research is required to determine the accurate mechanism.

mtDNA contains a unique 1,124-bp non-coding region, which is known as the displacement loop (D-loop). It has been observed that DNA methylation occurs in the D-loop region of mammals; however, D-loop regions of certain tumor tissue types were found to be de-methylated (9-11). Numerous studies have demonstrated that DNA hypomethylation is typically associated with gene activation. Furthermore, DNA hypomethylation also enhances DNA replication (12). However, whether DNA hypomethylation of the D-loop region of mtDNA is involved in the regulation of the mtDNA copy number and ND-2 expression in colorectal cancer has remained elusive. In the present study, the correlation between the methylation status of the D-loop region, mtDNA copy number and ND-2 expression was investigated in 65 colorectal cancers and their corresponding non-cancerous tissues. In addition, a de-methylation experiment was performed on the Caco-2 colorectal cancer cell line by using 5-aza-2'-deoxycytidine (5-Aza).

\section{Materials and methods}

Patients and specimens. Colorectal cancer tissues and their corresponding non-cancerous tissues were surgically resected from 30 colon- and 35 rectal cancer patients between November 2012 and January 2014 at West China Hospital, Sichuan University (Chengdu, China). This study was approved by the ethics committee of West China Hospital, Sichuan University (Chengdu, China). Informed consent was obtained from all patients. 36 of the patients were male and 25 were female (age, $35-70$ years; mean age, $53.8 \pm 12.3$ years). All specimens were immediately frozen in liquid nitrogen and stored at $-80^{\circ} \mathrm{C}$ for further DNA and protein analysis. Colorectal cancer tissues were resected from the edge of the tumors. In addition, their corresponding non-cancerous tissues at least $5 \mathrm{~cm}$ away from the tumors were also collected. The tumor, nodes and metastasis classification of malignant tumors (13) was applied to determine the clinicopathological stages of colorectal cancer by a senior pathologist.

Caco-2 cell culture and de-methylation experiment. The Caco-2 human colon adenocarcinoma cell line (Type Culture Collection of the Chinese Academy of Sciences, Shanghai, China) was cultured and maintained in Dulbecco's modified Eagle's medium (HyClone, Logan, UT, USA) in the presence of $10 \%$ fetal bovine serum (FBS; HyClone) supplemented with $100 \mathrm{U}$ penicillin and streptomycin (HyClone). Cells were cultured at $37^{\circ} \mathrm{C}$ in a humidified atmosphere containing $5 \%$ $\mathrm{CO}_{2}$. Caco-2 cells were serum-starved with $0.5 \% \mathrm{FBS}$ and then incubated with $5 \mathrm{mM}$ 5-Aza (Sigma-Aldrich, St. Louis, MO, USA) for $24 \mathrm{~h}$.

Methylation-specific polymerase chain reaction (MSP). mtDNA was extracted from specimens and cells by using the Mito isolation kit (GenMed Scientifics, Shanghai, China) according to the manufacturer's instructions. Methylation of the D-loop region of mtDNA was determined by MSP using bisulfite-modified DNA, prepared using an EpiTect Bisulfite kit (Qiagen, Dusseldorf, German), as previously described (9). Two primer sets were used to amplify the D-loop region of mtDNA that incorporated a number of $\mathrm{CpG}$ sites, one specific for the methylated sequence (D-loop-M forward, 5'-TGTTTCGGTTTTAGCGTTTC-3' and reverse, 5'-TACTACTCTCCTCGCTCCGA-3'); and the other for the unmethylated sequence (D-loop-U forward, 5'-GGGTGTTTTGGTTTTAGTGTTTT-3' and reverse, 5'-ATACTACTCTCCTCACTCCAAAC-3'). Primers were provided by Invitrogen Life Technologies, Carslbad, CA, USA). Universal methylated DNA (Qiagen) was used as a positive control and double-distilled $\mathrm{H}_{2} \mathrm{O}$ was served as a negative control. MSP assays were performed at least in duplicate.

Quantification of the mtDNA copy number. The relative mtDNA copy number was measured by real-time quantitative polymerase chain reaction (qPCR) as previously described (7). Briefly, two pairs of primers were employed, one primer pair was used for amplification of the mtDNA (forward, 5'-TACTCACCAGACGCCTCAACCG-3' and reverse, 5'-TTATCGGAATGGGAGGTGATTC-3'), and the other primer pair for the $\beta$-actin gene was run in parallel to standardize the input DNA (forward, 5'-CGGGAAATCGTGCGTGACAT-3' and reverse, 5'-GAAGGAAGGCTGGAAGAGTG-3'). The qPCR amplification was performed by using a SYBR Green qPCR kit (Bio-Rad Laboratories, Inc., Hercules, CA, USA) according to manufacturer's instructions on an Eppendorf Mastercycler ep realplex (Eppendorf, Hamburg, Germany). For the standard curve, an ultraviolet spectrophotometer (GE Healthcare Life Sciences, Piscataway, NJ, USA) was applied to quantify the initial concentration of the amplification products of the mtDNA and $\beta$-actin. Subsequently, the amplification products were serially diluted with a two-fold dilution to generate a six-point standard. Each measurement was performed in triplicate and a non-template control was included in each experiment.

Western blot analysis of ND-2 protein. Total protein was extracted from the tissues and cells by using a protein extraction kit (Nanjing Kaiji, Nanjing, China) following the manufacturer's instructions. After quantification by using the bicinchoninic acid assay (Nanjing Kaiji, Nanjing, China), $50 \mathrm{mg}$ proteins were separated by SDS-PAGE and transferred onto nitrocellulose membranes, which were incubated with rabbit anti-human polyclonal antibodies against ND-2 (1:400; cat. no. ab102753; Abcam, Cambridge, MA, USA) and cytochrome C oxidase subunit IV (Cox IV; 1:400; cat. no. bs-10257R; Bioss, Beijing, China) at $4^{\circ} \mathrm{C}$ overnight. The blots were washed and incubated with appropriate horseradish peroxidase-conjugated secondary antibodies (1:20,000; 
Table I. Association between methylation rate of the D-loop region and clinicopathological parameters in 65 colorectal cancer cases.

\begin{tabular}{|c|c|c|c|}
\hline \multirow[b]{2}{*}{ Characteristic } & \multirow[b]{2}{*}{ Number of cases } & \multicolumn{2}{|c|}{ Methylated D loop, n (\%) } \\
\hline & & Colorectal cancer & Corresponding non-cancerous \\
\hline Total & 65 & $9(13.8)$ & $53(81.5)$ \\
\hline \multicolumn{4}{|c|}{ Mean age (years) } \\
\hline$<50$ & 39 & $5(12.8)$ & $32(82.1)$ \\
\hline$\geq 50$ & 26 & $4(15.3)$ & $21(80.8)$ \\
\hline \multicolumn{4}{|l|}{ Gender } \\
\hline Male & 36 & $6(16.7)$ & $30(83.3)$ \\
\hline Female & 29 & $3(10.3)$ & $23(79.3)$ \\
\hline \multicolumn{4}{|c|}{ Clinicopathological stage } \\
\hline $\mathrm{I}$ & 16 & $4(25)$ & $13(81.3)$ \\
\hline II & 24 & $4(16.7)$ & $19(79.2)$ \\
\hline III & 14 & $1(7.1)$ & $12(85.7)$ \\
\hline IV & 11 & $0(0)$ & $9(81.8)$ \\
\hline
\end{tabular}

D-loop, displacement loop.

Santa Cruz Biotechnology, Inc., Santa Cruz, CA, USA) and visualized with an enhanced chemiluminescence detection kit (Amersham Pharmacia, Uppsala, Sweden). The autoradiographs were scanned following exposure of the membranes to Kodak XAR film (Kodak, Rochester, NY, USA). Protein expression was determined using Quantity One software 4.5.0 (Bio-Rad Laboratories, Inc.), and the protein expression levels were normalized to Cox IV.

Statistical analysis. All data are expressed as the mean \pm standard deviation and were analyzed using SPSS 13.0 software (SPSS, Inc., Chicago, IL, USA). Student's t-test was applied to compare differences in quantitative data. The $\chi^{2}$ test was applied to analyze differences between methylation rates and clinicopathological stages. $\mathrm{P}<0.05$ was considered to indicate a statistically significant difference between values.

\section{Results}

D-loop region is de-methylated in colorectal cancer. The methylation status and methylation rate of the D-loop region in the patient tissue samples, determined by the MSP assay, are listed in Table I. The results showed that the methylation rate of the D-loop region in all 65 colorectal cancer tissues was markedly reduced compared with that in their corresponding non-cancerous tissues (13.8 vs. 81.5\%; $\mathrm{P}<0.05)$. There was no significant correlation between the methylation rate of the D-loop region and the age or gender in colorectal cancer tissues and their corresponding non-cancerous tissues. However, the methylation rate of the D-loop region in the colorectal cancer tissues was markedly decreased in clinicopathological stages III and IV compared with stages I and II (7.1 and 0\% vs. 25 and 16\%; P<0.05). The methylation rate of the D-loop region in non-cancerous tissues was similar among the four clinicopathological stages $(81.3,79.2$, 85.7 and $81.8 \%$; $P>0.05)$.
mtDNA copy number is increased in colorectal cancer. qPCR was employed to quantify of the copy number of mtDNA in colorectal cancer tissues and their corresponding non-cancerous tissues. The relative mtDNA copy number was increased in $61(93.8 \%)$ colorectal cancer tissues when compared with that in their corresponding non-cancerous tissues (Fig. 1A). In addition, the mean relative mtDNA copy number in colorectal cancer tissues was increased when compared with that in the corresponding non-cancerous tissues $(127.2 \pm 34.3$ vs. $78.7 \pm 17.3$; $\mathrm{P}<0.05)$ (Fig. 1B).

ND-2 expression is upregulated in colorectal cancer. ND-2 protein in colorectal cancer tissues and their corresponding non-cancerous tissues was quantified by western blot analysis. The relative ND-2 protein content was increased in $58(89.2 \%)$ colorectal cancer tissues when compared with that in their corresponding non-cancerous tissues (Fig. 2A). In accordance with this result, the average relative ND-2 protein content of colorectal cancer tissues was also increased when compared with that in their corresponding non-cancerous tissues $(1.97 \pm 0.68$ vs. $1.03 \pm 0.39 ; \mathrm{P}<0.05)$ (Fig. $2 \mathrm{~B})$.

De-methylation of the D-loop region is associated with an increased mtDNA copy number. In order to characterize the manner in which the mtDNA copy number was altered, the correlation between the relative mtDNA copy number and the methylation status of the D-loop region was investigated in all 65 colorectal cancer tissues and their corresponding non-cancerous tissues. Among the 130 tissue samples, $68(52.3 \%)$ were unmethylated in the D-loop region, while $62(47.7 \%)$ tissues were methylated in the D-loop region. Furthermore, the relative mtDNA copy number was markedly elevated in the tissues with an unmethylated D-loop region when compared with the tissues with a methylated D-loop region $(128.8 \pm 29.7$ vs. $76.4 \pm 14.6$; $\mathrm{P}<0.05)$, as shown in Fig. 3A. Subsequently, the present study evaluated the 
A

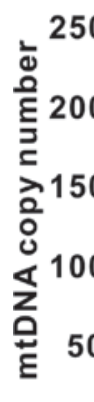

(1)
Colorectal cancer tissues

Corresponding non-cancerous tissues

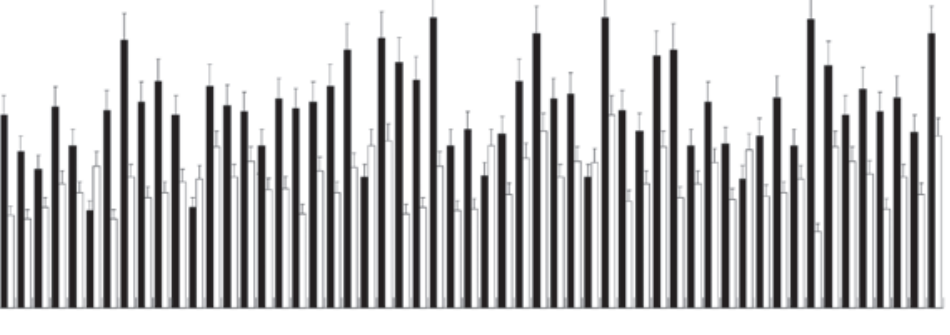

11
31
41

51

61
B

Colorectal cancer tissues

Corresponding non-cancerous tissues

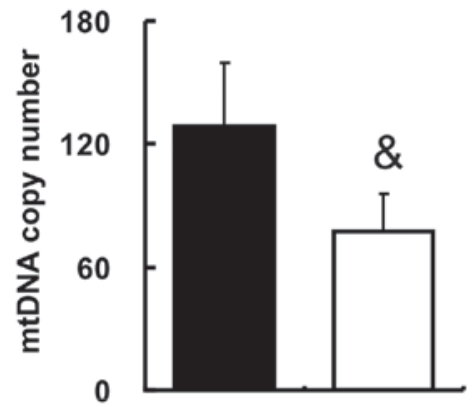

Figure 1. mtDNA copy number in colorectal cancer tissues and corresponding non-cancerous tissues. (A) Relative mtDNA copy number in colorectal cancer tissues and their corresponding non-cancerous tissues in each case. (B) Comparison of the average relative mtDNA copy number between the 65 cases of colorectal cancer tissues and their corresponding non-cancerous tissues. ${ }^{\circledR} \mathrm{P}<0.05$ vs. colorectal cancer tissues. mtDNA, mitochondrial DNA.

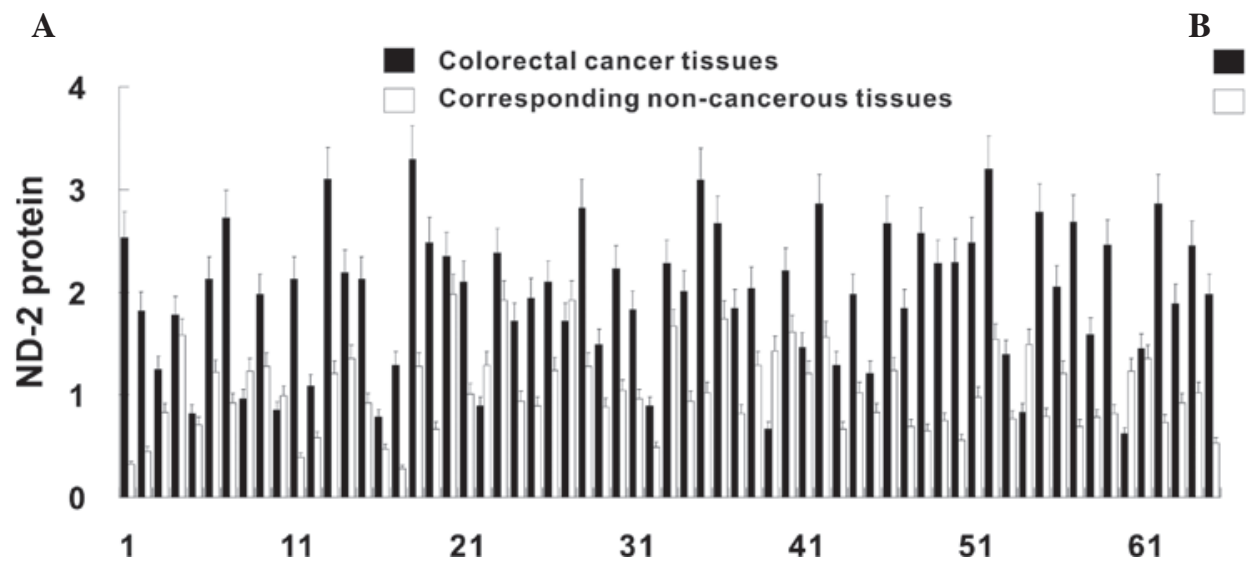

Colorectal cancer tissues

Corresponding non-cancerous tissues

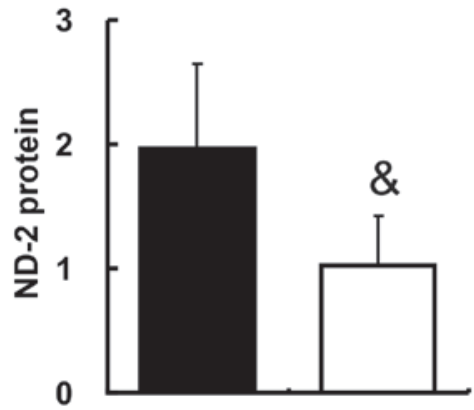

Figure 2. ND-2 expression in colorectal cancer tissues and corresponding non-cancerous tissues. (A) Relative ND-2 expression of colorectal cancer tissues and their corresponding non-cancerous tissues in each case. (B) Comparison of the average relative ND-2 expression between the 65 cases of colorectal cancer tissues and their corresponding non-cancerous tissues. ${ }^{\circledR} \mathrm{P}<0.05$ vs. colorectal cancer tissues. ND-2, nicotinamide adenine dinucleotide subunit 2.

A

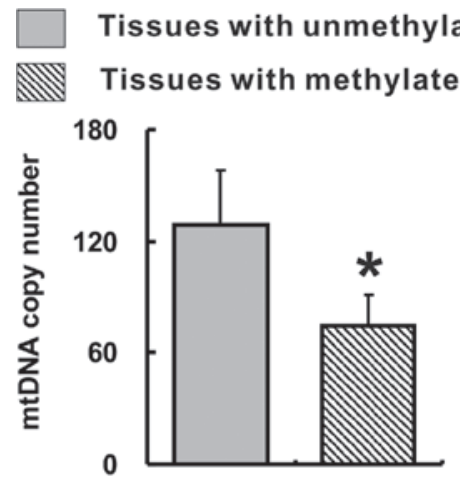



Figure 3. De-methylation of the D-loop region is associated with increased mtDNA copy number. (A) Comparison of the average relative mtDNA copy number between tissues of 68 unmethylated and 62 methylated D-loop regions. (B) Representative gel of the PCR products of three replicate de-methylation experiments of the Caco-2 cells; (C) Average relative mtDNA copy number in vehicle- and 5-Aza-treated Caco-2 cells. "P<0.05 vs. tissues with unmethylated D-loop; ${ }^{\text {"P}} \mathrm{P}<0.05$ vs. vehicle-treated Caco-2. 5-Aza, 5-aza-2'-deoxycytidine; $\mathrm{U}$, unmethylated; M, methylated; mtDNA, mitochondrial DNA.

effect of de-methylation of the D-loop region in vitro. The MSP results confirmed that treatment with 5-Aza altered the D-loop region from a methylation to a de-methylation status (Fig. 3B). Consequently, the relative mtDNA copy number was significantly increased in the 5-Aza-treated Caco-2 cells when compared with that measured in the vehicle-treated
Caco- 2 cells $(90.2 \pm 13.1$ vs. $115.4 \pm 19.8 ; \mathrm{P}<0.05)$, as shown in Fig. 3C.

De-methylation of the D-loop region is associated with increased $N D-2$ expression. The correlation between relative ND-2 expression and methylation status of the D-loop region 
A

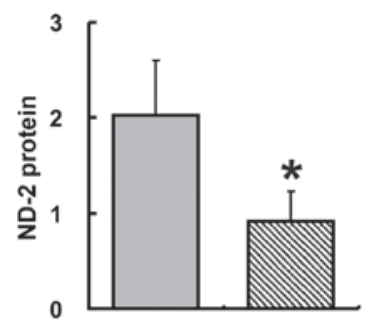

B

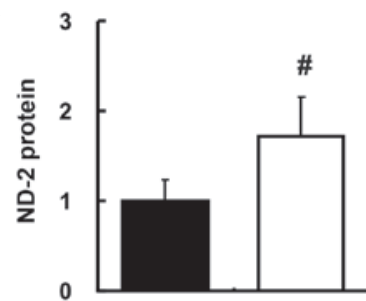

C

ND-2

Cox IV

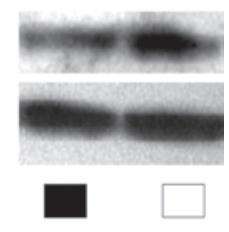

Vehicle

5-Aza

Figure 4. De-methylation of the D-loop region is associated with increased ND-2 expression. (A) Comparison of the average relative ND-2 expression between tissues of 68 unmethylated and 62 methylated D-loop regions. (B) Average relative ND-2 expression in vehicle- and 5-Aza-treated Caco-2 cells. (C) Representative blot from 65 repetitions of the de-methylation experiment on Caco-2 cells; ND-2 and Cox IV protein levels were quantified by western blot analysis. " $\mathrm{P}<0.05$ vs. tissues with unmethylated D-loop region; " $\mathrm{P}<0.05$ vs. vehicle treatment Caco-2. ND-2, nicotinamide adenine dinucleotide subunit 2 ; Cox IV, cytochrome C oxidase subunit IV; 5-Aza, 5-aza-2'-deoxycytidine.

was also investigated. The ND-2 expression was significantly increased in tissues with an unmethylated D-loop region when compared to that in tissues with a methylated D-loop region $(2.03 \pm 0.58$ vs. $0.92 \pm 0.31 ; \mathrm{P}<0.05)$ (Fig. 4A). Furthermore, the ND-2 expression was conspicuously increased in 5-Aza-treated Caco- 2 cells when compared with that in the vehicle-treated Caco- 2 cells $(1.00 \pm 0.23$ vs. $1.72 \pm 0.43 ; \mathrm{P}<0.05)$ (Fig. 4B and C).

Elevated mtDNA copy number is correlated with increased ND-2 expression. The correlation between the mtDNA copy number and the relative ND-2 expression in colorectal cancer tissues and their corresponding non-cancerous tissues was investigated by using a general linear correlation model. A positive linear correlation between mtDNA copy number and relative ND-2 expression was observed $(\mathrm{R}=0.685, \mathrm{P}<0.05)$ (Fig. 5), which indicated that increased ND-2 expression was correlated with an elevated mtDNA copy number.

\section{Discussion}

Abnormal epigenetic alterations of several nuclear DNA-encoded oncogenes have been suggested to be involved in driving the tumorigenesis of colorectal cancer (14); however, little attention has been paid to the involvement of mtDNA alternation in the pathobiology of colorectal cancer. The present study demonstrated that de-methylation of the D-loop region elevated the mtDNA copy number and that the expression of ND-2 was concomitantly increased in colorectal cancer tissues; furthermore, an association of the methylation status of the D-loop region with the clinicopathological stage was established in colorectal cancer tissues. In addition, the de-methylation of the D-loop region was associated with an elevated mtDNA copy number and an increased ND-2 expression in tissue samples as well as in the in vitro cell experiment.

The epigenetic modification of the D-loop region regulates the replication and the transcription of mtDNA $(10,15)$. Methylation, one of the most important types of epigenetic modification, occurs at the $\mathrm{CpG}$ islands and is typically associated with gene silencing (12). The present study manifested that the de-methylation rate of the D-loop region in colorectal cancer tissues was significantly higher than that in the

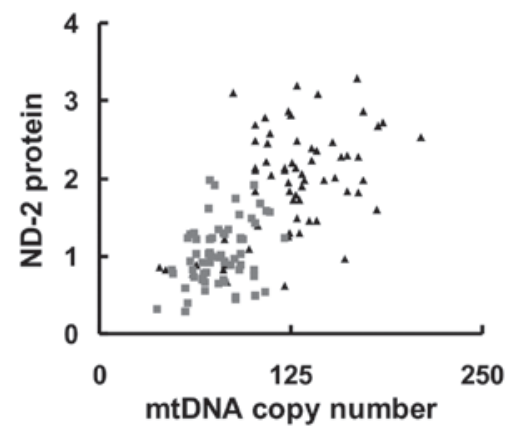

\section{Colorectal cancer tissues}

A Corresponding non-cancerous tissues

Figure 5. Relative ND-2 expression correlates linearly with the mtDNA copy number. Elevated mtDNA copy number was associated with increased expression of ND-2 during the development of colorectal cancer. ND-2, nicotinamide adenine dinucleotide subunit 2; mtDNA, mitochondrial DNA.

corresponding non-cancerous tissues. Furthermore, the D-loop region was predominantly de-methylated in samples with clinicopathological stages I and II (25 and 16.7\%, respectively), while it was largely or completely de-methylated in samples with clinicopathological stages III and IV (7.1 and 0\%). The gradient of the de-methylation rate from clinicopathological stages I-IV suggested that the de-methylation of the D-loop region increases during the progression of colorectal cancer. These findings are consistent with the results of a previous study, which indicated that the de-methylation of the D-loop region is an early molecular event in the genesis of colorectal cancer (9).

In post-mitotic cells, mtDNA replicates continuously, leading to an increases in the mtDNA copy number (16); however, the mechanisms of mtDNA replication have remained to be fully elucidated. The D-loop, a non-coding region of the mtDNA, is important for the regulation of mitochondrial genome replication and expression, as it contains the initial site of heavy-chain replication and the promoters for heavy- and light-chain transcription (17). Mutation or epigenetic modifications in the D-loop region may affect mtDNA replication and change the expression pattern of mtDNA $(10,15,18,19)$. The present study manifested that de-methylation of the D-loop 
region was associated with an elevated mtDNA copy number and increased ND-2 expression in colorectal cancer tissues as well as in their corresponding non-cancerous tissues. Of note, the mtDNA copy number and ND-2 expression were significantly increased after 5-Aza treatment. These findings suggested that de-methylation of the D-loop region is an important factor that regulates mtDNA replication and ND-2 expression in colorectal cancer; however, the exact mechanism remains elusive.

It has been gradually established that mtDNA maintenance protein mitochondrial transcription factor A (TFAM) regulates mtDNA levels $(16,20)$. TFAM stimulates transcription and replication of mtDNA by binding to the D-loop region (21). However, methylation of the $\mathrm{CpG}$ islands of the D-loop region inhibits transcription factor binding, consequently suppressing transcription and replication of mtDNA. In the present study, methylation of the D-loop region was observed in $13.8 \%$ of colorectal cancer tissues and $81.5 \%$ of their corresponding non-cancerous tissues. Furthermore, de-methylation of the $\mathrm{D}$-loop region resulted in elevation of the mtDNA copy number and in induction of ND-2 expression in vitro. From these results, it can be presumed that de-methylation of the D-loop region facilitates TFAM binding and eventually promotes the replication and transcription of mtDNA. However, further study is required to verify this hypothesis.

Cancer cells require higher levels of energy than normal cells to sustain their rapid proliferation. The energy is mainly supplied by oxidative phosphorylation in the mitochondria $(22,23)$. ND-2 is a subunit of NADH which is involved in oxidative phosphorylation. In the present study, upregulation of ND-2 was observed in colorectal cancer tissues compared with that in their corresponding non-cancerous tissues. Presumably, the increase of ND-2 gives rise to enhanced oxidative phosphorylation in colorectal cancer. In accord with this result, it was demonstrated by a recent study that circulating cancer cells exhibit enhanced mitochondrial biogenesis and respiration, as well as up-regulation of genes associated with oxidative phosphorylation (22). Furthermore, the relative ND-2 expression was linearly correlated with the mtDNA copy number, which suggested that the elevated mtDNA copy number together with the de-methylation of the D-loop region may be involved in the up-regulation of ND-2 expression.

In conclusion, in colorectal cancer tissues, the de-methylation rate of the D-loop region, the mtDNA copy number and ND-2 expression were significantly higher than those in the corresponding non-cancerous tissues. De-methylation of the D-loop region may be involved in the regulation of the mtDNA copy number and ND-2 expression.

\section{Acknowledgements}

The present study was supported by a grant from the Natural Science Fund of China (no. 81301770). The authors would like to thank the doctors from the Gastrointestinal Surgery Department of West China Hospital (Chengdu, China) for collecting the specimens.

\section{References}

1. Siegel R, Desantis C and Jemal A: Colorectal cancer statistics, 2014. CA Cancer J Clin 64: 104-117, 2014.

2. Center MM, Jemal A, Smith RA and Ward E: Worldwide variations in colorectal cancer. CA Cancer J Clin 59: 366-378, 2009.

3. Grivell LA: Mitochondrial DNA. Small, beautiful and essential. Nature 341: 569-571, 1989.

4. Lightowlers RN, Chinnery PF, Turnbull DM and Howell N: Mammalian mitochondrial genetics: Heredity, heteroplasmy and disease. Trends Genet 13: 450-455, 1997.

5. Zhang G, Qu Y, Dang S, Yang Q, Shi B and Hou P: Variable copy number of mitochondrial DNA (mtDNA) predicts worse prognosis in advanced gastric cancer patients. Diagn Pathol 8: 173, 2013.

6. Cheau-Feng Lin F, Jeng YC, Huang TY, Chi CS, Chou MC and Chin-Shaw Tsai S: Mitochondrial DNA copy number is associated with diagnosis and prognosis of head and neck cancer. Biomarkers 19: 269-274, 2014.

7. Feng S, Xiong L, Ji Z, Cheng W and Yang H: Correlation between increased copy number of mitochondrial DNA and clinicopathological stage in colorectal cancer. Oncol Lett 2: 899-903, 2011.

8. Boland ML, Chourasia AH and Macleod KF: Mitochondrial dysfunction in cancer. Front Oncol 3: 292, 2013.

9. Feng S, Xiong L, Ji Z, Cheng W and Yang H: Correlation between increased ND2 expression and demethylated displacement loop of mtDNA in colorectal cancer. Mol Med Rep 6: 125-130, 2012.

10. Bellizzi D, D'Aquila P, Scafone T, Giordano M, Riso V, Riccio A and Passarino G: The control region of mitochondrial DNA shows an unusual $\mathrm{CpG}$ and non-CpG methylation pattern. DNA Res 20: 537-547, 2013.

11. Roos-Araujo D, Stuart S, Lea RA, Haupt LM and Griffiths LR: Epigenetics and migraine; complex mitochondrial interactions contributing to disease susceptibility. Gene 543: 1-7, 2014.

12. Esteller M: $\mathrm{CpG}$ island hypermethylation and tumor suppressor genes: A booming present, a brighter future. Oncogene 21: 5427-5440, 2002.

13. Mates IN, Jinga V, Csiki IE, Mates D, Dinu D, Constantin A and Jinga M: Single nucleotide polymorphisms in colorectal cancer: associations with tumor site and TNM stage. J Gastrointestin Liver Dis 21: 45-52, 2012.

14. Matsubara N: Epigenetic regulation and colorectal cancer. Dis Colon Rectum 55: 96-104, 2012.

15. Zhang R, Zhang F, Wang C, Wang S, Shiao YH and Guo Z: Identification of sequence polymorphism in the D-Loop region of mitochondrial DNA as a risk factor for hepatocellular carcinoma with distinct etiology. J Exp Clin Cancer Res 29: 130, 2010.

16. Ylikallio E, Tyynismaa H, Tsutsui H, Ide T and Suomalainen A: High mitochondrial DNA copy number has detrimental effects in mice. Hum Mol Genet 19: 2695-2705, 2010.

17. Penta JS, Johnson FM, Wachsman JT and Copeland WC: Mitochondrial DNA in human malignancy. Mutat Res 488: 119-133, 2001.

18. Zhang J, Guo Z, Bai Y, Cui L, Zhang S and Xu J: Identification of sequence polymorphisms in the displacement loop region of mitochondrial DNA as a risk factor for renal cell carcinoma. Biomed Rep 1: 563-566, 2013.

19. Tommasi S, Favia P, Weigl S, Bianco A, Pilato B, Russo L, Paradiso A and Petruzzella V: Mitochondrial DNA variants and risk of familial breast cancer: An exploratory study. Int J Oncol 44: 1691-1698, 2014.

20. Maniura-Weber K, Goffart S, Garstka HL, Montoya J and Wiesner RJ: Transient overexpression of mitochondrial transcription factor A (TFAM) is sufficient to stimulate mitochondrial DNA transcription, but not sufficient to increase mtDNA copy number in cultured cells. Nucleic Acids Res 32: 6015-6027, 2004.

21. Kurita T, Izumi H, Kagami S, Kawagoe T, Toki N, Matsuura Y, Hachisuga T and Kohno K: Mitochondrial transcription factor A regulates BCL2L1 gene expression and is a prognostic factor in serous ovarian cancer. Cancer Sci 103: 239-444, 2012.

22. LeBleu VS, O'Connell JT, Gonzalez Herrera KN, Wikman H, Pantel K, Haigis MC, de Carvalho FM, Damascena A, Domingos Chinen LT, Rocha RM, et al: PGC- $1 \alpha$ mediates mitochondrial biogenesis and oxidative phosphorylation in cancer cells to promote metastasis. Nat Cell Biol 16: 992-1003, 2014.

23. Lim SH, Wu L, Kiew LV, Chung LY, Burgess K and Lee HB: Rosamines targeting the cancer oxidative phosphorylation pathway. PloS one 9: e82934, 2014. 